\title{
Measuring Higgs boson couplings at the CERN LHC
}

\author{
D. Zeppenfeld, ${ }^{1}$ R. Kinnunen, ${ }^{2}$ A. Nikitenko, ${ }^{2, *}$ and E. Richter-Wa̧s ${ }^{3}$ \\ ${ }^{1}$ Department of Physics, University of Wisconsin, Madison, Wisconsin 53706 \\ and CERN, TH Division, 1211 Geneva 23, Switzerland \\ ${ }^{2}$ Helsinki Institute of Physics, Helsinki, Finland \\ ${ }^{3}$ CERN, IT Division, 1211 Geneva 23, Switzerland \\ and Institute of Computer Science, Jagellonian University, Institute of Nuclear Physics, \\ 30-055 Krakow, ul. Kawiory 26a, Poland \\ (Received 3 February 2000; published 8 June 2000)
}

\begin{abstract}
For an intermediate mass Higgs boson with SM-like couplings the CERN LHC allows observation of a variety of decay channels in production by gluon fusion and weak boson fusion. Cross section ratios provide measurements of various ratios of Higgs couplings, with accuracies of order $15 \%$ for $100 \mathrm{fb}^{-1}$ of data in each of the two LHC experiments. For Higgs boson masses above $120 \mathrm{GeV}$, minimal assumptions on the Higgs sector allow for an indirect measurement of the total Higgs boson width with an accuracy of 10 to $20 \%$, and of the $H \rightarrow W W$ partial width with an accuracy of about $10 \%$.

PACS number(s): 14.80.Bn, 13.85.-t
\end{abstract}

\section{INTRODUCTION}

Investigation of the symmetry breaking mechanism of the electroweak $S U(2) \times U(1)$ gauge symmetry will be one of the prime tasks of the CERN Large Hadron Collider (LHC). Correspondingly, major efforts have been concentrated on devising methods for Higgs boson discovery, for the entire mass range allowed within the standard model (SM) (100 $\mathrm{GeV} \lesssim m_{H} \lesssim 1 \mathrm{TeV}$, after the CERN $e^{+} e^{-}$collider LEP2), and for Higgs boson search in extensions of the SM, such as the minimal supersymmetric standard model (MSSM) [1,2]. While observation of one or more Higgs scalar(s) at the LHC appears assured, discovery will be followed by a more demanding task: the systematic investigation of Higgs boson properties. Beyond observation of the various $C P$ even and $C P$ odd scalars which nature may have in store for us, this means the determination of the couplings of the Higgs boson to the known fermions and gauge bosons, i.e. the measurement of $H t t, H b b, H \tau \tau$ and $H W W, H Z Z, H \gamma \gamma$ couplings, to the extent possible.

Clearly this task very much depends on the expected Higgs boson mass. For $m_{H}>200 \mathrm{GeV}$ and within the SM, only the $H \rightarrow Z Z$ and $H \rightarrow W W$ channels are expected to be observable, and the two gauge boson modes are related by $\mathrm{SU}(2)$. Above $m_{H} \approx 250 \mathrm{GeV}$, where detector effects will no longer dominate the mass resolution of the $H \rightarrow Z Z \rightarrow 4 l$ resonance, additional information is expected from a direct measurement of the total Higgs boson width, $\Gamma_{H}$. A much richer spectrum of decay modes is predicted for the intermediate mass range, i.e. if a SM-like Higgs boson has a mass between the reach of LEP2 $(\lesssim 110 \mathrm{GeV})$ and the Z-pair threshold. The main reasons for focusing on this range are present indications from electroweak precision data, which favor $m_{H}<250 \mathrm{GeV}$ [3], as well as expectations within the MSSM, which predicts the lightest Higgs boson to have a mass $m_{h} \lesssim 130 \mathrm{GeV}$ [4].

\footnotetext{
*On leave from ITEP, Moscow, Russia.
}

Until recently, the prospects of detailed and model independent coupling measurements at the LHC were considered somewhat remote [5], because few promising search channels were known to be accessible, for any given Higgs boson mass. Taking ATLAS search scenarios as an example, these were [1]

$$
\begin{aligned}
& g g \rightarrow H \rightarrow \gamma \gamma, \text { for } m_{H} \lesssim 150 \mathrm{GeV}, \\
& g g \rightarrow H \rightarrow Z Z^{*} \rightarrow 4 l, \text { for } m_{H} \gtrsim 130 \mathrm{GeV},
\end{aligned}
$$

and

$$
g g \rightarrow H \rightarrow W W^{*} \rightarrow l \bar{\nu} \bar{l} \nu, \quad \text { for } m_{H} \gtrsim 150 \mathrm{GeV}
$$

with the possibility of obtaining some additional information from processes like $W H$ and/or $t \bar{t} H$ associated production with subsequent $H \rightarrow \bar{b} b$ and $H \rightarrow \gamma \gamma$ decay for Higgs boson masses near $100 \mathrm{GeV}$. Throughout this paper, " $g g \rightarrow H$ ", stands for inclusive Higgs production, which is dominated by the gluon fusion process for a SM-like Higgs boson.

This relatively pessimistic outlook is changing considerably now, due to the demonstration that weak boson fusion is a promising Higgs production channel also in the intermediate mass range. Previously, this channel had only been explored for Higgs boson masses above $300 \mathrm{GeV}$. Specifically, it was recently shown in parton level analyses that the weak boson fusion channels, with subsequent Higgs boson decay into photon pairs $[6,7]$ :

$$
q q \rightarrow q q H, \quad H \rightarrow \gamma \gamma, \quad \text { for } m_{H} \lesssim 150 \mathrm{GeV}
$$

into $\tau^{+} \tau^{-}$pairs [7-9],

$$
q q \rightarrow q q H, \quad H \rightarrow \tau \tau, \quad \text { for } m_{H} \lesssim 140 \mathrm{GeV},
$$

or into $W$ pairs $[7,10]$ 


$$
\begin{gathered}
q q \rightarrow q q H, \quad H \rightarrow W W^{(*)} \rightarrow e^{ \pm} \mu^{\mp} p_{T}, \\
\text { for } m_{H} \gtrsim 120 \mathrm{GeV},
\end{gathered}
$$

can be isolated at the LHC. Preliminary analyses, which try to extend these parton level results to full detector simulations, look promising [11]. The weak boson fusion channels utilize the significant background reductions which are expected from double forward jet tagging [12-14] and central jet vetoing techniques $[15,16]$, and promise low background environments in which Higgs boson decays can be studied in detail. The parton level results predict highly significant signals with (substantially) less than $100 \mathrm{fb}^{-1}$.

The prospect of observing several Higgs production and decay channels, over the entire intermediate mass range, suggests a reanalysis of coupling determinations at the LHC [5]. In this paper we attempt a first such analysis, for the case where the branching fractions of an intermediate mass Higgs resonance are fairly similar to the SM case, i.e. we analyze a SM-like Higgs boson only. We make use of the previously published analyses for the inclusive Higgs production channels $[1,2]$ and of the weak boson fusion channels [6-10]. The former were obtained by the experimental collaborations and include detailed detector simulations. The latter are based on parton level results, which employ full QCD tree level matrix elements for all signal and background processes. We will not discuss here differences in the performance expected for the ATLAS and CMS detectors nor details in the theoretical assumptions which lead to different estimates for expected signal and background rates. The reader is referred to the original publications from which numbers are extracted. In Sec. II we summarize expectations for the various channels, including expected accuracies for cross section measurement of the various signals for an integrated luminosity of $100 \mathrm{fb}^{-1}$. Implications for the determination of coupling ratios and the measurement of Higgs boson (partial) decay widths are then obtained in Sec. III. A final summary is given in Sec. IV.

\section{SURVEY OF INTERMEDIATE MASS HIGGS CHANNELS}

The various Higgs channels listed in Eqs. (1)-(6) and their observability at the LHC have all been discussed in the literature. Where available, we give values as presently quoted by the experimental collaborations. In order to compare the accuracy with which the cross sections of different Higgs production and decay channels can be measured, we need to unify these results. For example, $K$-factors of unity are assumed throughout. Our goal in this section is to obtain reasonable estimates for the relative errors, $\Delta \sigma_{H} / \sigma_{H}$, which are expected after collecting $100 \mathrm{fb}^{-1}$ in each the ATLAS and the CMS detector, i.e. we estimate results after a total of $200 \mathrm{fb}^{-1}$ of data have been collected at the LHC. Presumably these data will be taken with a mix of both low and high luminosity running.

We find that the measurements are largely dominated by statistical errors. For all channels, event rates with $200 \mathrm{fb}^{-1}$ of data will be large enough to use the Gaussian approximation for statistical errors. The experiments measure the signal cross section by separately determining the combined signal + background rate, $N_{S+B}$, and the expected number of background events, $\left\langle N_{B}\right\rangle$. The signal cross section is then given by

$$
\sigma_{H}=\frac{N_{S+B}-\left\langle N_{B}\right\rangle}{\epsilon \int \mathcal{L} d t}=\frac{N_{S}}{\epsilon \int \mathcal{L} d t},
$$

where $\epsilon$ denotes efficiency factors. Thus the statistical error is given by

$$
\frac{\Delta \sigma_{H}}{\sigma_{H}}=\frac{\sqrt{N_{S+B}}}{N_{S}}=\frac{\sqrt{N_{S}+N_{B}}}{N_{S}},
$$

where in the last step we have dropped the distinction between the expected and the actual number of background events. Systematic errors on the background rate are added in quadrature to the background statistical error, $\sqrt{N_{B}}$, where appropriate.

Well below the $H \rightarrow W W$ threshold, the search for $H$ $\rightarrow \gamma \gamma$ events is arguably the cleanest channel for Higgs discovery. LHC detectors have been designed for excellent twophoton invariant mass resolution, with this Higgs signal in mind. We directly take the expected signal and background rates for the inclusive $H \rightarrow \gamma \gamma$ search from the detailed studies of the CMS and ATLAS collaborations [17,18,1], which were performed for an integrated luminosity of $100 \mathrm{fb}^{-1}$ in each detector. Expectations are summarized in Table I. Rates correspond to not including a $K$-factor for the expected signal and background cross sections in CMS and ATLAS. Cross sections have been determined with MRS (R1) parton distribution functions (pdf's) for CMS, while ATLAS numbers are based on CTEQ2L pdf's.

The inclusive $H \rightarrow \gamma \gamma$ signal will be observed as a narrow $\gamma \gamma$ invariant mass peak on top of a smooth background distribution. This means that the background can be directly measured from the very high statistics background distribution in the sidebands. We expect any systematic errors on the extraction of the signal event rate to be negligible compared to the statistical errors which are given in the last row of Table I. With $100 \mathrm{fb}^{-1}$ of data per experiment $\sigma(g g$ $\rightarrow H) \cdot B(H \rightarrow \gamma \gamma)$ can be determined with a relative error of 10 to $15 \%$ for Higgs boson masses between 100 and 150 $\mathrm{GeV}$. Here we do not include additional systematic errors, e.g. from the luminosity uncertainty or from higher order QCD corrections, because we will mainly consider cross section ratios in the final analysis in the next section. These systematic errors largely cancel in the cross section ratios. Systematic errors common to several channels will be considered later, where appropriate.

A Higgs search channel with a much better signal to background ratio, at the price of lower statistics, however, is available via the inclusive search for $H \rightarrow Z Z^{*} \rightarrow 4 l$ events. Expected event numbers for $100 \mathrm{fb}^{-1}$ in both ATLAS [1] and CMS [19] are listed in Table II. These numbers were derived using CTEQ2L pdf's and are corrected to contain no QCD $K$-factor. For those Higgs boson masses where no ATLAS or CMS prediction is available, we interpolate- 
TABLE I. Number of expected events for the inclusive SM $H \rightarrow \gamma \gamma$ signal and expected backgrounds, assuming an integrated luminosity of $100 \mathrm{fb}^{-1}$ and high luminosity performance. Numbers correspond to optimal $\gamma \gamma$ invariant mass windows for CMS and ATLAS. The expected relative statistical errors on the signal cross section are given for the individual experiments and are combined in the last line.

\begin{tabular}{|c|c|c|c|c|c|c|c|}
\hline & $m_{H}$ & 100 & 110 & 120 & 130 & 140 & 150 \\
\hline \multirow[t]{3}{*}{ CMS $[17,18]$} & $N_{S}$ & 865 & 1038 & 1046 & 986 & 816 & 557 \\
\hline & $N_{B}$ & 29120 & 22260 & 16690 & 12410 & 9430 & 7790 \\
\hline & $\Delta \sigma_{H} / \sigma_{H}$ & $20.0 \%$ & $14.7 \%$ & $12.7 \%$ & $11.7 \%$ & $12.4 \%$ & $16.4 \%$ \\
\hline \multirow[t]{3}{*}{ ATLAS [1] } & $N_{S}$ & 1045 & 1207 & 1283 & 1186 & 973 & 652 \\
\hline & $N_{B}$ & 56450 & 47300 & 39400 & 33700 & 28250 & 23350 \\
\hline & $\Delta \sigma_{H} / \sigma_{H}$ & $22.9 \%$ & $18.2 \%$ & $15.7 \%$ & $15.7 \%$ & $17.6 \%$ & $23.8 \%$ \\
\hline Combined & $\Delta \sigma_{H} / \sigma_{H}$ & $15.1 \%$ & $11.4 \%$ & $9.9 \%$ & $9.4 \%$ & $10.1 \%$ & $13.5 \%$ \\
\hline
\end{tabular}

extrapolate the results for the nearest Higgs boson mass, taking the expected $H \rightarrow Z Z^{*}$ branching ratios into account for the signal. Similar to the case of $H \rightarrow \gamma \gamma$ events, the signal is seen as a narrow peak in the four-lepton invariant mass distribution, i.e. the background can be extracted directly from the signal sidebands. The combined relative error on the measurement of $\sigma(g g \rightarrow H) \cdot B\left(H \rightarrow Z Z^{*}\right)$ is listed in the last line of Table II. For Higgs boson masses in the 130-150 $\mathrm{GeV}$ range, and above $Z$-pair threshold, a $10 \%$ statistical error on the cross section measurement is possible. In the intermediate range, where $H \rightarrow W W$ dominates, and for lower Higgs boson masses, where the Higgs boson is expected to dominantly decay into $\bar{b} b$, the error increases substantially.

Above $m_{H} \approx 135 \mathrm{GeV}, H \rightarrow W W^{(*)}$ becomes the dominant SM Higgs boson decay channel. The resulting inclusive $W W \rightarrow l^{+} \nu l^{-} \bar{\nu}$ signal is visible above backgrounds, after exploiting the characteristic lepton angular correlations for spin zero decay into $W$ pairs near threshold [20]. The inclusive channel, which is dominated by $g g \rightarrow H \rightarrow W W$, has been analyzed by ATLAS for $m_{H} \geqslant 150 \mathrm{GeV}$ and for integrated luminosities of 30 and $100 \mathrm{fb}^{-1}$ [1] and by CMS for $m_{H}$ $\geqslant 120 \mathrm{GeV}$ and $30 \mathrm{fb}^{-1}$ [20]. The expected event numbers for $30 \mathrm{fb}^{-1}$ are listed in Table III. The numbers are derived without QCD K-factors and use CTEQ2L for ATLAS and MRS(A) pdf's for CMS results.

Unlike the two previous modes, the two missing neutrinos in the $H \rightarrow W W$ events do not allow for a reconstruction of the narrow Higgs boson mass peak. Since the Higgs signal is only seen as a broad enhancement of the expected background rate in lepton-neutrino transverse mass distributions, with similar shapes of signal and background after application of all cuts, a precise determination of the background rate from the data is not possible. Rather one has to rely on background measurements in phase space regions where the signal is weak, and extrapolation to the search region using NLO QCD predictions. The precise error on this extrapolation is unknown at present, the assumption of a 5\% systematic background uncertainty appears optimistic but attainable. It turns out that with $30 \mathrm{fb}^{-1}$ already, the systematic error starts to dominate, because the background exceeds the signal rate by factors of up to 5 , depending on the Higgs boson mass. Running at high luminosity makes matters worse, because the less efficient reduction of $\bar{t} t$ backgrounds,

TABLE II. Number of expected events for the inclusive SM $H \rightarrow Z Z^{*} \rightarrow l^{+} l^{-} l^{+} l^{-}$signal and expected backgrounds, assuming an integrated luminosity of $100 \mathrm{fb}^{-1}$ and high luminosity performance. Numbers correspond to optimal four-lepton invariant mass windows for CMS and ATLAS and to the combined total. Rates in parentheses correspond to numbers interpolated, according to $H \rightarrow Z Z^{*}$ branching ratios for the signal. The expected relative statistical errors on the signal cross section are given for each experiment and are combined in the last line.

\begin{tabular}{llrrrrrrr}
\hline \hline & \multicolumn{1}{c}{$m_{H}$} & 120 & 130 & 140 & 150 & 160 & 170 & 180 \\
\hline CMS [19] & $N_{S}$ & 19.2 & 55.3 & $(99)$ & 131.4 & $(48)$ & 29.4 & $(76.5)$ \\
& $N_{B}$ & 12.9 & 17.1 & $(20)$ & 22.5 & $(26)$ & 27.5 & $(27)$ \\
& $\Delta \sigma_{H} / \sigma_{H}$ & $29.5 \%$ & $15.4 \%$ & $11.0 \%$ & $9.4 \%$ & $17.9 \%$ & $25.7 \%$ & $13.3 \%$ \\
& & & & & & & & \\
ATLAS [1] & $N_{S}$ & 10.3 & 28.7 & $(51)$ & 67.6 & $(31)$ & 19.1 & 49.7 \\
& $N_{B}$ & 4.44 & 7.76 & $(8)$ & 8.92 & $(8)$ & 8.87 & 8.81 \\
& $\Delta \sigma_{H} / \sigma_{H}$ & $37.3 \%$ & $21.0 \%$ & $15.1 \%$ & $12.9 \%$ & $20.1 \%$ & $27.7 \%$ & $15.4 \%$ \\
& & & & & & & & $13 \%$ \\
Combined & $\Delta \sigma_{H} / \sigma_{H}$ & $23.1 \%$ & $12.4 \%$ & $8.9 \%$ & $7.6 \%$ & $13.4 \%$ & $18.8 \%$ & $10.1 \%$ \\
\hline \hline
\end{tabular}


TABLE III. Number of expected events for the inclusive SM $H \rightarrow W W^{*} \rightarrow l^{+} \nu l^{-} \bar{\nu}$ signal and expected backgrounds, assuming an integrated luminosity of $30 \mathrm{fb}^{-1}$. Numbers correspond to optimized cuts, varying with the mass of the Higgs boson being searched for. The expected relative errors on the signal cross section are given for each experiment, separating the statistical error, the effect of a systematic 5\% error of the background level, and the two added in quadrature. The combined error for the two experiments assumes $100 \%$ correlation of the systematic errors on the background determination.

\begin{tabular}{llrrrrrrrr}
\hline \hline \multicolumn{1}{c}{$m_{H}$} & 120 & 130 & 140 & 150 & 160 & 170 & 180 & 190 \\
\hline CMS [20] & $N_{S}$ & 44 & 106 & 279 & 330 & 468 & 371 & 545 & \\
& $N_{B}$ & 272 & 440 & 825 & 732 & 360 & 360 & 1653 & \\
& $\Delta \sigma_{H} / \sigma_{H}$ (stat.) & $40.4 \%$ & $22.0 \%$ & $11.9 \%$ & $9.9 \%$ & $6.1 \%$ & $7.3 \%$ & $8.6 \%$ & \\
& $\Delta \sigma_{H} / \sigma_{H}$ (syst.) & $30.9 \%$ & $20.8 \%$ & $14.8 \%$ & $11.1 \%$ & $3.8 \%$ & $4.9 \%$ & $15.2 \%$ & \\
& $\Delta \sigma_{H} / \sigma_{H}$ (comb.) & $50.9 \%$ & $30.3 \%$ & $19.0 \%$ & $14.9 \%$ & $7.3 \%$ & $8.8 \%$ & $17.4 \%$ & $20.6 \%$ \\
& & & & & & & & & \\
ATLAS [1] & $N_{S}$ & & & & 240 & 400 & 337 & 276 & 124 \\
& $N_{B}$ & & & & 844 & 656 & 484 & 529 & 301 \\
& $\Delta \sigma_{H} / \sigma_{H}$ (stat.) & & & & $13.7 \%$ & $8.1 \%$ & $8.5 \%$ & $10.3 \%$ & $16.6 \%$ \\
& $\Delta \sigma_{H} / \sigma_{H}$ (syst.) & & & & & & & & \\
& $\Delta \sigma_{H} / \sigma_{H}$ (comb.) & $50.9 \%$ & $30.3 \%$ & $19.0 \%$ & $22.3 \%$ & $11.5 \%$ & $11.1 \%$ & $14.1 \%$ & $20.6 \%$ \\
& & & & & & & & & \\
Combined & $\Delta \sigma_{H} / \sigma_{H}$ (comb.) & $42.1 \%$ & $26.0 \%$ & $17.0 \%$ & $14.8 \%$ & $7.0 \%$ & $8.0 \%$ & $13.6 \%$ & $16.9 \%$ \\
\hline \hline
\end{tabular}

due to less stringent $b$-jet veto criteria, increases the background rate further. Because of this problem we only present results for $30 \mathrm{fb}^{-1}$ of low luminosity running in Table III. Since neither of the LHC Collaborations has presented predictions for the entire Higgs boson mass range, we take CMS simulations below $150 \mathrm{GeV}$ and ATLAS results at $190 \mathrm{GeV}$, but divide the resultant statistical errors by a factor $\sqrt{2}$, to take account of the presence of two experiments. Between 150 and $180 \mathrm{GeV}$ we combine both experiments, assuming $100 \%$ correlation in the systematic $5 \%$ normalization error of the background.

The previous analyses are geared towards measurement of the inclusive Higgs production cross section, which is dominated by the gluon fusion process. $15-20 \%$ of the signal sample, however, is expected to arise from weak boson fusion, $q q \rightarrow q q H$ or corresponding antiquark initiated processes. The weak boson fusion component can be isolated by making use of the two forward tagging jets which are present in these events and by vetoing additional central jets, which are unlikely to arise in the color singlet signal process [15]. A more detailed discussion of these processes can be found in Ref. [7] from which most of the following numbers are taken.

The $q q \rightarrow q q H, H \rightarrow \gamma \gamma$ process was first analyzed in Ref. [6], where cross sections for signal and background were obtained with full QCD tree level matrix elements. The parton level Monte Carlo determines all geometrical acceptance corrections. Additional detector effects were included by smearing parton and photon 4-momenta with expected detector resolutions and by assuming trigger, identification and reconstruction efficiencies of 0.86 for each of the two tagging jets and 0.8 for each photon. Resulting cross sections were presented in Ref. [7] for a fixed $\gamma \gamma$ invariant mass window of total width $\Delta m_{\gamma \gamma}=2 \mathrm{GeV}$. We correct these

TABLE IV. Number of expected $\gamma \gamma j j$ events from the $q q \rightarrow q q H, H \rightarrow \gamma \gamma$ weak boson fusion signal and expected backgrounds, assuming an integrated luminosity of $100 \mathrm{fb}^{-1}$. Numbers correspond to optimal $\gamma \gamma$ invariant mass windows for CMS and ATLAS and to the combined total, as projected from the parton level analysis of Refs. [6,7]. The expected relative statistical errors on the signal cross section are given for each experiment and are combined in the last line.

\begin{tabular}{|c|c|c|c|c|c|c|c|}
\hline & $m_{H}$ & 100 & 110 & 120 & 130 & 140 & 150 \\
\hline \multirow{3}{*}{$\begin{array}{l}\text { Projected CMS } \\
\text { performance }\end{array}$} & $N_{S}$ & 37 & 48 & 56 & 56 & 48 & 33 \\
\hline & $N_{B}$ & 33 & 32 & 31 & 30 & 28 & 25 \\
\hline & $\Delta \sigma_{H} / \sigma_{H}$ & $22.6 \%$ & $18.6 \%$ & $16.7 \%$ & $16.6 \%$ & $18.2 \%$ & $23.1 \%$ \\
\hline \multirow{3}{*}{$\begin{array}{l}\text { Projected ATLAS } \\
\text { performance }\end{array}$} & $N_{S}$ & 42 & 54 & 63 & 63 & 54 & 37 \\
\hline & $N_{B}$ & 61 & 60 & 56 & 54 & 51 & 46 \\
\hline & $\Delta \sigma_{H} / \sigma_{H}$ & $24.2 \%$ & $19.8 \%$ & $17.3 \%$ & $17.2 \%$ & $19.0 \%$ & $24.6 \%$ \\
\hline Combined & $\Delta \sigma_{H} / \sigma_{H}$ & $16.5 \%$ & $13.6 \%$ & $12.0 \%$ & $11.9 \%$ & $13.1 \%$ & $16.8 \%$ \\
\hline
\end{tabular}


TABLE V. Number of expected signal and background events for the $q q \rightarrow q q H \rightarrow \tau \tau j j$ channel, for $100 \mathrm{fb}^{-1}$ and two detectors. Cross sections are added for $\tau \tau \rightarrow l^{ \pm} h^{\mp} p_{T}$, i.e., hadronic decay of one of the $\tau^{\prime}$ s, and $\tau \tau \rightarrow e^{ \pm} \mu^{\mp} p_{T}$ events, as given in Refs. [7,9]. The last line gives the expected statistical relative error on the $q q$ $\rightarrow q q H, H \rightarrow \tau \tau$ cross section.

\begin{tabular}{lrrrrrr}
\hline \hline \multicolumn{1}{c}{$m_{H}$} & 100 & 110 & 120 & 130 & 140 & 150 \\
\hline$N_{S}$ & 211 & 197 & 169 & 128 & 79 & 38 \\
$N_{B}$ & 305 & 127 & 51 & 32 & 27 & 24 \\
$\Delta \sigma_{H} / \sigma_{H}$ & $10.8 \%$ & $9.1 \%$ & $8.8 \%$ & $9.9 \%$ & $13.0 \%$ & $20.7 \%$ \\
\hline \hline
\end{tabular}

numbers for $m_{H}$ dependent mass resolutions in the experiments. We take $1.4 \sigma$ mass windows, as given in Ref. [1] for high luminosity running, which are expected to contain $79 \%$ of the signal events for ATLAS. The $2 \mathrm{GeV}$ window for $m_{H}=100 \mathrm{GeV}$ at CMS $[17,18]$ is assumed to scale up like the ATLAS resolution and assumed to contain $70 \%$ of the Higgs signal. The expected total signal and background rates for $100 \mathrm{fb}^{-1}$ and resulting relative errors for the extraction of the signal cross section are given in Table IV. Statistical errors only are considered for the background subtraction, since the background level can be measured independently by considering the sidebands to the Higgs boson peak.

The next weak boson fusion channel to be considered is $q q \rightarrow q q H, H \rightarrow \tau \tau$. Again, this channel has been analyzed at the parton level, including some estimates of detector effects, as discussed for the $H \rightarrow \gamma \gamma$ case. Here, a lepton identification efficiency of 0.95 is assumed for each lepton $l=e, \mu$. Two $\tau$-decay modes have been considered so far: $H$ $\rightarrow \tau \tau \rightarrow l^{ \pm} h^{\mp} p_{T}[8]$ where $h^{ \pm}$stands for $\pi^{ \pm}, \rho^{ \pm}$or $a_{1}^{ \pm}$, and $H \rightarrow \tau \tau \rightarrow e^{ \pm} \mu^{+} p_{T}$ [9]. These analyses were performed for low luminosity running. Some deterioration at high luminosity is expected, as in the analogous $H / A \rightarrow \tau \tau$ channel in the MSSM search [1]. At high luminosity, pile-up effects degrade the $p_{T}$ resolution significantly, which results in a worse $\tau \tau$ invariant mass resolution. At a less significant level, a higher $p_{T}$ threshold for the minijet veto technique will increase the QCD and $t \bar{t}$ backgrounds. The $\tau$-identification efficiency is similar at high and low luminosity. We expect that the reduced performance at high luminosity can be compensated for by considering the additional channels $H$ $\rightarrow \tau \tau \rightarrow e^{+} e^{-} p_{T}, \mu^{+} \mu^{-} p_{T}$. Z+ jets and $Z Z+$ jets backgrounds (with $Z Z \rightarrow l^{+} l^{-} \nu \bar{\nu}$ ) are strongly suppressed by rejecting same flavor lepton pairs which are compatible with $Z$ decays $\left(m_{l l}=m_{Z} \pm 6 \mathrm{GeV}\right)$. Drell-Yan plus jets backgrounds are further reduced by requiring significant $p_{T}$. Since these analyses have not yet been performed, we use the predicted cross sections for only those two channels which have already been discussed in the literature and scale event rates to a combined $200 \mathrm{fb}^{-1}$ of data. Results are given in Table V.

The previous two weak boson fusion channels allow reconstruction of the Higgs resonance as an invariant mass peak. This is not the case for $H \rightarrow W W \rightarrow l^{+} \nu l^{-} \bar{\nu}$ as discussed previously for the inclusive search. The weak boson fusion channel can be isolated separately by employing forward jet tagging and color singlet exchange isolation tech-
TABLE VI. Number of events expected for $q q \rightarrow q q H, H$ $\rightarrow W W^{(*)} \rightarrow \mu^{ \pm} e^{\mp} p_{T}$ in $200 \mathrm{fb}^{-1}$ of data, and corresponding backgrounds [10]. The expected relative statistical error on the signal cross section is given in the last line.

\begin{tabular}{lccccrrrr}
\hline \hline$m_{H}$ & 120 & 130 & 140 & 150 & 160 & 170 & 180 & 190 \\
\hline$N_{S}$ & 136 & 332 & 592 & 908 & 1460 & 1436 & 1172 & 832 \\
$N_{B}$ & 136 & 160 & 188 & 216 & 240 & 288 & 300 & 324 \\
$\Delta \sigma_{H} / \sigma_{H}$ & $12.1 \%$ & $6.7 \%$ & $4.7 \%$ & $3.7 \%$ & $2.8 \%$ & $2.9 \%$ & $3.3 \%$ & $4.1 \%$ \\
\hline \hline
\end{tabular}

niques in addition to tools like charged lepton angular correlations which are used for the inclusive channel. The corresponding parton level analysis for $q q \rightarrow q q H, H$ $\rightarrow W W^{(*)} \rightarrow \mu^{ \pm} e^{\mp} \boldsymbol{p}_{T}$ has been performed in Ref. [10] and we here scale the results to a total integrated luminosity of $200 \mathrm{fb}^{-1}$, which takes into account the availability of two detectors. As for the tau case, the analysis was done for low luminosity running conditions and somewhat higher backgrounds are expected at high luminosity. On the other hand the $W W^{(*)} \rightarrow \mu^{+} \mu^{-} p_{T}$ and $W W^{(*)} \rightarrow e^{+} e^{-} p_{T}$ modes should roughly double the available statistics since very few signal events have lepton pair invariant masses compatible with $Z$ $\rightarrow l l$ decays. Therefore our estimates are actually conservative. Note that the expected background for this weak boson fusion process is much smaller than for the corresponding inclusive measurement. As a result modest systematic uncertainties will not degrade the accuracy with which $\sigma(q q$ $\rightarrow q q H) \cdot B\left(H \rightarrow W W^{(*)}\right)$ can be measured (see Table VI). A $10 \%$ systematic error on the background, double the error assumed in the inclusive case, would degrade the statistical accuracy by, typically, a factor 1.2 or less. As a result, we expect that a very precise measurement of $\sigma(q q$ $\rightarrow q q H) \cdot B\left(H \rightarrow W W^{(*)}\right)$ can be performed at the LHC, with a statistical accuracy of order 5\% or even better in the mass range $m_{H} \geqslant 140 \mathrm{GeV}$. Even for $m_{H}$ as low as $120 \mathrm{GeV}$ a $12 \%$ measurement is expected.

\section{MEASUREMENT OF HIGGS PROPERTIES}

One would like to translate the cross section measurements of the various Higgs production and decay channels into measurements of Higgs boson properties, in particular into measurements of the various Higgs boson couplings to gauge fields and fermions. This translation requires knowledge of NLO QCD corrections to production cross sections, information on the total Higgs decay width and a combination of the measurements discussed previously. The task here is to find a strategy for combining the anticipated LHC data without undue loss of precision due to theoretical uncertainties and systematic errors.

For our further discussion it is convenient to rewrite all Higgs boson couplings in terms of partial widths of various Higgs boson decay channels. The Higgs-fermion couplings $g_{H f f}$, for example, which in the SM are given by the fermion masses, $g_{H f f}=m_{f}\left(m_{H}\right) / v$, can be traded for the $H \rightarrow \bar{f} f$ partial widths: 
TABLE VII. Summary of the accuracy with which various ratios of partial widths can be determined with $200 \mathrm{fb}^{-1}$ of data. The first two columns give the ratio considered and indicate the method by which it is measured. $Y_{Z} / Y_{W}$, for example, indicates a measurement of $\sigma B\left(H \rightarrow Z Z^{*}\right) / \sigma B\left(H \rightarrow W W^{*}\right)$ in gluon fusion, while $X_{i}$ ratios correspond to weak boson fusion (see text for details). The statistical combination of several channels for a given width ratio is indicated by $\oplus .5 \%$ and $20 \%$ theoretical uncertainties for weak boson and gluon fusion cross sections affect the mixed gluon-weak boson fusion ratios only, which are needed for a measurement of $\Gamma_{g} / \Gamma_{W}$. The effect of this systematic error is indicated in the last line.

\begin{tabular}{|c|c|c|c|c|c|c|c|c|c|c|}
\hline$m_{H}$ & & 100 & 110 & 120 & 130 & 140 & 150 & 160 & 170 & 180 \\
\hline \multirow[t]{4}{*}{$z=\Gamma_{Z} / \Gamma_{W}$} & $Y_{Z} / Y_{W}$ & & & $48 \%$ & $29 \%$ & $19 \%$ & $17 \%$ & $15 \%$ & $20 \%$ & $17 \%$ \\
\hline & $Y_{Z} X_{\gamma}$ & & & $30 \%$ & $21 \%$ & $19 \%$ & $23 \%$ & & & \\
\hline & $\overline{Y_{\gamma}} \overline{X_{W}}$ & & & & & & & & & \\
\hline & $\frac{Y_{Z}}{Y_{W}} \oplus \frac{Y_{Z}}{Y_{\gamma}} \frac{X_{\gamma}}{X_{W}}$ & & & $29 \%$ & $19 \%$ & $15 \%$ & $14 \%$ & $15 \%$ & $20 \%$ & $17 \%$ \\
\hline$\Gamma_{\gamma} / \Gamma_{W}$ & $\frac{Y_{\gamma}}{Y_{W}} \oplus \frac{X_{\gamma}}{X_{W}}$ & & & $16 \%$ & $12 \%$ & $11 \%$ & $13 \%$ & & & \\
\hline$\Gamma_{\tau} / \Gamma_{W}$ & $\frac{X_{\tau}}{X_{W}}$ & & & $15 \%$ & $12 \%$ & $14 \%$ & $21 \%$ & & & \\
\hline$\Gamma_{\tau} / \Gamma_{\gamma}$ & $\frac{X_{\tau}}{X_{\gamma}}$ & $20 \%$ & $16 \%$ & $15 \%$ & $16 \%$ & $18 \%$ & $27 \%$ & & & \\
\hline \multirow[t]{2}{*}{$\Gamma_{g} / \Gamma_{W}$} & $\frac{Y_{\gamma}}{X_{\gamma}} \oplus \frac{Y_{W}}{X_{W}}$ & $22 \%$ & $18 \%$ & $15 \%$ & $13 \%$ & $12 \%$ & $13 \%$ & $8 \%$ & $9 \%$ & $14 \%$ \\
\hline & $\frac{Y_{\gamma}}{X_{\gamma}} \oplus \frac{Y_{W}}{X_{W}} \oplus 21 \%$ & $30 \%$ & $27 \%$ & $25 \%$ & $24 \%$ & $24 \%$ & $24 \%$ & $22 \%$ & $22 \%$ & $25 \%$ \\
\hline
\end{tabular}

$$
\Gamma_{f}=\Gamma(H \rightarrow \bar{f} f)=c_{f} \frac{g_{H f f}^{2}}{8 \pi}\left(1-\frac{4 m_{f}^{2}}{m_{H}^{2}}\right)^{3 / 2} m_{H} .
$$

Here $c_{f}$ is the color factor (1 for leptons, 3 for quarks). Similarly the square of the $H W W$ coupling $\left(g_{H W W}=g m_{W}\right.$ in the $\mathrm{SM})$ or the $H Z Z$ coupling is proportional to the partial widths $\Gamma_{W}=\Gamma\left(H \rightarrow W W^{*}\right)$ or $\Gamma_{Z}=\Gamma\left(H \rightarrow Z Z^{*}\right) \quad[21]$. Analogously we trade the squares of the effective $H \gamma \gamma$ and $H g g$ couplings for $\Gamma_{\gamma}=\Gamma(H \rightarrow \gamma \gamma)$ and $\Gamma_{g}=\Gamma(H \rightarrow g g)$. Note that the $H g g$ coupling is essentially proportional to $g_{H t t}$, the Higgs' coupling to the top quark.

The Higgs production cross sections are governed by the same squares of couplings. This allows to write e.g. the $g g$ $\rightarrow H$ production cross section as [22]

$$
\sigma(g g \rightarrow H)=\Gamma(H \rightarrow g g) \frac{\pi^{2}}{8 m_{H}^{3}} \tau \int_{\tau}^{1} \frac{d x}{x} g\left(x, m_{H}^{2}\right) g\left(\frac{\tau}{x}, m_{H}^{2}\right),
$$

where $\tau=m_{H}^{2} / s$. Similarly the $q q \rightarrow q q H$ cross sections via $W W$ and $Z Z$ fusion are proportional to $\Gamma\left(H \rightarrow W W^{*}\right)$ and $\Gamma\left(H \rightarrow Z Z^{*}\right)$, respectively. In the narrow width approximation, which is appropriate for the intermediate Higgs boson mass range considered here, these production cross sections need to be multiplied by the branching fractions for final state $j, B(H \rightarrow j)=\Gamma_{j} / \Gamma$, where $\Gamma$ denotes the total Higgs width. This means that the various cross section measure- ments discussed in the previous section provide measurements of various combinations $\Gamma_{i} \Gamma_{j} / \Gamma$.

The production cross sections are subject to QCD corrections, which introduces theoretical uncertainties. While the $K$-factor for the gluon fusion process is large [23], which suggests a sizable theoretical uncertainty on the production cross section, the NLO corrections to the weak boson fusion cross section are essentially identical to the ones encountered in deep inelastic scattering and are quite small [24]. Thus we can assign a small theoretical uncertainty to the latter, of order 5\%, while we shall use a larger theoretical error for the gluon fusion process, of order 20\% [23]. The problem for weak boson fusion is that it consists of a mixture of $Z Z$ $\rightarrow H$ and $W W \rightarrow H$ events, and we cannot distinguish between the two experimentally. In a large class of models the ratio of $H W W$ and $H Z Z$ couplings is identical to the one in the SM, however, and this includes the MSSM. We therefore make the following $W, Z$-universality assumption:

(1) The $H \rightarrow Z Z^{*}$ and $H \rightarrow W W^{*}$ partial widths are related by $\mathrm{SU}(2)$ as in the $\mathrm{SM}$, i.e. their ratio, $z$, is given by the SM value,

$$
\Gamma_{Z}=z \Gamma_{W}=z_{S M} \Gamma_{W}
$$

Note that this assumption can be tested, at the 15-20\% level for $m_{H}>130 \mathrm{GeV}$, by forming the ratio $B \sigma(g g$ $\left.\rightarrow H \rightarrow Z Z^{*}\right) / B \sigma\left(g g \rightarrow H \rightarrow W W^{*}\right)$, in which QCD uncertainties cancel (see Table VII). Here $B$ indicates the branch- 
ing fraction of the Higgs boson in a given decay channel.

With $W, Z$-universality, the three weak boson fusion cross sections give us direct measurements of three combinations of (partial) widths,

$$
\begin{gathered}
X_{\gamma}=\frac{\Gamma_{W} \Gamma_{\gamma}}{\Gamma} \text { from } q q \rightarrow q q H, \quad H \rightarrow \gamma \gamma, \\
X_{\tau}=\frac{\Gamma_{W} \Gamma_{\tau}}{\Gamma} \quad \text { from } q q \rightarrow q q H, \quad H \rightarrow \tau \tau, \\
X_{W}=\frac{\Gamma_{W}^{2}}{\Gamma} \quad \text { from } q q \rightarrow q q H, \quad H \rightarrow W W^{(*)},
\end{gathered}
$$

with common theoretical systematic errors of $5 \%$. In addition the three gluon fusion channels provide measurements of

$$
\begin{gathered}
Y_{\gamma}=\frac{\Gamma_{g} \Gamma_{\gamma}}{\Gamma} \quad \text { from } g g \rightarrow H \rightarrow \gamma \gamma, \\
Y_{Z}=\frac{\Gamma_{g} \Gamma_{Z}}{\Gamma} \text { from } g g \rightarrow H \rightarrow Z Z^{(*)}, \\
Y_{W}=\frac{\Gamma_{g} \Gamma_{W}}{\Gamma} \quad \text { from } g g \rightarrow H \rightarrow W W^{(*)},
\end{gathered}
$$

with common theoretical systematic errors of $20 \%$.

The first precision test of the Higgs sector is provided by taking ratios of the $X_{i}$ 's and ratios of the $Y_{i}$ 's. In these ratios the QCD uncertainties, and all other uncertainties related to the initial state, like luminosity and pdf errors, cancel. Beyond testing $W, Z$-universality, these ratios provide useful information for Higgs boson masses between 100 and 150 $\mathrm{GeV}$ and 120 to $150 \mathrm{GeV}$, respectively, where more than one channel can be observed in the weak boson fusion and gluon fusion groups. Typical errors on these cross section ratios are expected to be in the 15 to $20 \%$ range (see Table VII). Accepting an additional systematic error of about $20 \%$, a measurement of the ratio $\Gamma_{g} / \Gamma_{W}$ can be performed, by measuring the cross section ratios $B \sigma(g g \rightarrow H \rightarrow \gamma \gamma) / \sigma(q q$ $\rightarrow q q H) B(H \rightarrow \gamma \gamma) \quad$ and $\quad B \sigma\left(g g \rightarrow H \rightarrow W W^{*}\right) / \sigma(q q$ $\rightarrow q q H) B\left(H \rightarrow W W^{*}\right)$. Expected accuracies are listed in Table VII. In these estimates the systematics coming from understanding detector acceptance is not included.

Within the SM, the quark triangle diagrams which lead to an effective $H g g$ coupling are dominated by the top-quark loop. In this limit $\Gamma_{g} / \Gamma_{W}$ determines the Htt to $H W W$ coupling ratio. The underlying assumption of top-dominance may not be realized in nature, e.g. if the $H b b$ Yukawa coupling is exceptionally large. However, such a scenario would either result in a strongly reduced rate for the $H \rightarrow \tau \tau$ signal or, when the $H \tau \tau$ coupling is increased proportional to $g_{H b b}$, the ratio $\Gamma_{\tau} / \Gamma_{W}$ would be increased substantially. In many cases, the measured values of various ratios in Table VII would alert us to caution in interpreting measurements involving the $\mathrm{Hgg}$ vertex in terms of the top-quark Yukawa coupling alone. Completely new contributions to the $\mathrm{Hg}$ coupling, from as yet unknown heavy colored particles, cannot be distinguished from the top-quark triangle, of course.

Beyond the measurement of coupling ratios, minimal additional assumptions allow an indirect measurement of the total Higgs width. First of all, the $\tau$ partial width, properly normalized, is measurable with an accuracy of order $10 \%$. The $\tau$ is a third generation fermion with isospin $-\frac{1}{2}$, just like the $b$-quark. In all extensions of the SM with a common source of lepton and quark masses, even if generational symmetry is broken, the ratio of $b$ to $\tau$ Yukawa couplings is given by the fermion mass ratio. We thus assume, in addition to $W, Z$-universality, that

(2) The ratio of $b$ to $\tau$ couplings of the Higgs boson is given by their mass ratio, i.e.

$$
y=\frac{\Gamma_{b}}{\Gamma_{\tau}}=3 c_{Q C D} \frac{g_{H b b}^{2}}{g_{H \tau \tau}^{2}}=3 c_{Q C D} \frac{m_{b}^{2}\left(m_{H}\right)}{m_{\tau}^{2}},
$$

where $c_{Q C D}$ is the known QCD and phase space correction factor.

(3) The total Higgs width is dominated by decays to $\bar{b} b$, $\tau \tau, W W, Z Z, g g$ and $\gamma \gamma$, i.e. the branching ratio for unexpected channels is small:

$$
\begin{aligned}
\epsilon= & 1-\left(B(H \rightarrow b \bar{b})+B(H \rightarrow \tau \tau)+B\left(H \rightarrow W W^{(*)}\right)\right. \\
& \left.+B\left(H \rightarrow Z Z^{(*)}\right)+B(H \rightarrow g g)+B(H \rightarrow \gamma \gamma)\right) \\
\ll & 1 .
\end{aligned}
$$

Note that, in the Higgs boson mass range of interest, these two assumptions are satisfied for both $C P$ even Higgs bosons in most of the MSSM parameter space. The first assumption holds in the MSSM at tree level, but can be violated by large squark loop contributions, in particular for small $m_{A}$ and large $\tan \beta[25,26]$. The second assumption might be violated, for example, if the $H \rightarrow \bar{c} c$ partial width is exceptionally large. However, a large up-type Yukawa coupling would be noticeable in the $\Gamma_{g} / \Gamma_{W}$ coupling ratio, which measures the Htt coupling.

With these assumptions consider the observable

$$
\begin{aligned}
\widetilde{\Gamma}_{W} & =X_{\tau}(1+y)+X_{W}(1+z)+X_{\gamma}+\tilde{X}_{g} \\
& =\left(\Gamma_{\tau}+\Gamma_{b}+\Gamma_{W}+\Gamma_{Z}+\Gamma_{\gamma}+\Gamma_{g}\right) \frac{\Gamma_{W}}{\Gamma} \\
& =(1-\epsilon) \Gamma_{W},
\end{aligned}
$$

where $\widetilde{X}_{g}=\Gamma_{g} \Gamma_{W} / \Gamma$ is determined by combining $Y_{W}$ and the product $Y_{\gamma} X_{W} / X_{\gamma} \cdot \widetilde{\Gamma}_{W}$ provides a lower bound on $\Gamma(H$ $\left.\rightarrow W W^{(*)}\right)=\Gamma_{W}$. Provided $\epsilon$ is small $(\epsilon<0.1$ suffices for practical purposes), the determination of $\widetilde{\Gamma}_{W}$ provides a direct measurement of the $H \rightarrow W W^{(*)}$ partial width. Once $\Gamma_{W}$ has been determined, the total width of the Higgs boson is given by 


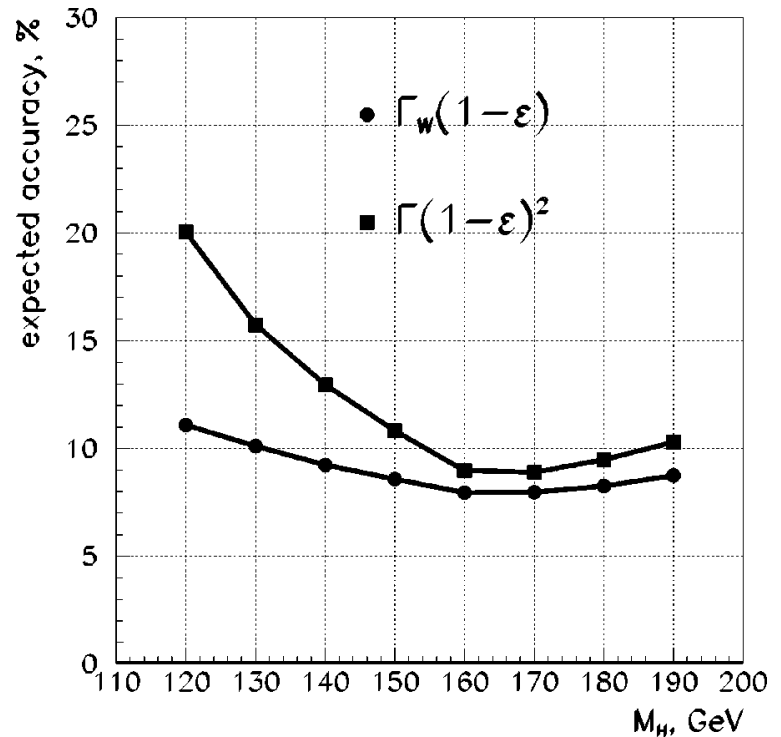

FIG. 1. Expected accuracy with which the Higgs boson width can be measured at the LHC, with $100 \mathrm{fb}^{-1}$ of data in each experiment. Results are shown for the extraction of the $H \rightarrow W W$ partial with, $\Gamma_{W}$, and the total Higgs boson width, $\Gamma . \epsilon$ is the sum of the residual (small) branching ratios of unobserved channels, mainly $H \rightarrow c \bar{c}$ (see text for detail).

$$
\Gamma=\frac{\Gamma_{W}^{2}}{X_{W}}=\frac{1}{X_{W}}\left(X_{\tau}(1+y)+X_{W}(1+z)+X_{\gamma}+\tilde{X}_{g}\right)^{2} \frac{1}{(1-\epsilon)^{2}} .
$$

For a SM-like Higgs boson the Higgs width is dominated by the $H \rightarrow \bar{b} b$ and $H \rightarrow W W^{(*)}$ channels. Thus, the error on $\widetilde{\Gamma}_{W}$ is dominated by the uncertainties of the $X_{W}$ and $X_{\tau}$ measurements and by the theoretical uncertainty on the $b$-quark mass, which enters the determination of $y$ quadratically. According to the Particle Data Group, the present uncertainty on the $b$ quark mass is about $\pm 3.5 \%$ [27]. Assuming a luminosity error of $\pm 5 \%$ in addition to the theoretical uncertainty of the weak boson fusion cross section of $\pm 5 \%$, the statistical errors of the $q q \rightarrow q q H, H \rightarrow \tau \tau$ and $q q \rightarrow q q H, H$ $\rightarrow W W$ cross sections of Tables V and VI lead to an expected accuracy of the $\widetilde{\Gamma}_{W}$ determination of order $10 \%$. More precise estimates, as a function of the Higgs boson mass, are shown in Fig. 1.

The extraction of the total Higgs width, via Eq. (21), requires a measurement of the $q q \rightarrow q q H, H \rightarrow W W^{(*)}$ cross section, which is expected to be available for $m_{H} \gtrsim 115 \mathrm{GeV}$ [10]. Consequently, errors are large for Higgs boson masses close to this lower limit (we expect a relative error of $\approx 20 \%$ for $m_{H}=120 \mathrm{GeV}$ and $\left.\epsilon<0.05\right)$. But for Higgs boson masses around the $W W$ threshold, $\Gamma(1-\epsilon)^{2}$ can be determined with an error of about $10 \%$. Results are shown in Fig. 1 and look highly promising.

\section{SUMMARY}

In the last section we have found that various ratios of Higgs partial widths can be measured with accuracies of or- der 10 to $20 \%$, with an integrated luminosity of $100 \mathrm{fb}^{-1}$ per experiment. This translates into 5 to $10 \%$ measurements of various ratios of coupling constants. The ratio $\Gamma_{\tau} / \Gamma_{W}$ measures the coupling of down-type fermions relative to the Higgs couplings to gauge bosons. To the extent that the $H \gamma \gamma$ triangle diagrams are dominated by the $W$ loop, the width ratio $\Gamma_{\tau} / \Gamma_{\gamma}$ measures the same relationship. The fermion triangles leading to an effective $H g g$ coupling are expected to be dominated by the top-quark, thus, $\Gamma_{g} / \Gamma_{W}$ probes the coupling of up-type fermions relative to the $H W W$ coupling. Finally, for Higgs boson masses above $\approx 120 \mathrm{GeV}$, the absolute normalization of the $H W W$ coupling is accessible via the extraction of the $H \rightarrow W W^{(*)}$ partial width in weak boson fusion.

Note that these measurements test the crucial aspects of the Higgs sector. The $H W W$ coupling, being linear in the Higgs field, identifies the observed Higgs boson as the scalar responsible for the spontaneous breaking of $S U(2) \times U(1)$ : a scalar without a vacuum expectation value couples to gauge bosons only via $H H W W$ or $H H W$ vertices at tree level, i.e. the interaction is quadratic in scalar fields. The absolute value of the $H W W$ coupling, as compared to the SM expectation, reveals whether $H$ may be the only mediator of spontaneous symmetry breaking or whether additional Higgs bosons await discovery. Within the framework of the MSSM this is a measurement of $|\sin (\beta-\alpha)|$, at the \pm 0.05 level. The measurement of the ratios of $g_{H t t} / g_{H W W}$ and $g_{H \tau \tau} / g_{H W W}$ then probes the mass generation of both up and down type fermions.

The results presented here constitute a first look only at the issue of coupling extractions for the Higgs boson. This is the case for the weak boson fusion processes in particular, which prove to be extremely valuable if not essential. Our analysis is mostly an estimate of statistical errors, with some rough estimates of the systematic errors which are to be expected for the various measurements of (partial) widths and their ratios. A number of issues need to be addressed in further studies, in particular with regard to the weak boson fusion channels.

(a) The weak boson fusion channels and their backgrounds have only been studied at the parton level, to date. Full detector level simulations, and optimization of strategies with more complete detector information is crucial for further progress.

(b) A central jet veto has been suggested as a powerful tool to suppress QCD backgrounds to the color singlet exchange processes which we call weak boson fusion. The feasibility of this tool and its reach need to be investigated in full detector studies, at both low and high luminosity.

(c) In the weak boson fusion studies of $H \rightarrow W W$ and $H$ $\rightarrow \tau \tau$ decays, double leptonic $e^{+} e^{-} p_{T}$ and $\mu^{+} \mu^{-} p_{T}$ signatures have not yet been considered. Their inclusion promises to almost double the statistics available for the Higgs coupling measurements, at the price of additional $Z Z+$ jets and Drell-Yan plus jets backgrounds which are expected to be manageable.

(d) Other channels, like $W H$ or $t \bar{t} H$ associated production with subsequent decay $H \rightarrow \bar{b} b$ or $H \rightarrow \gamma \gamma$, provide addi- 
tional information on Higgs coupling ratios, which complement our analysis at small Higgs boson mass values, $m_{H}$ $\lesssim 120 \mathrm{GeV}[2,5]$. These channels need to be included in the analysis.

(e) Much additional work is needed on more reliable background determinations. For the $H \rightarrow W W^{(*)} \rightarrow l^{+} l^{\prime-} p_{T}$ channel in particular, where no narrow Higgs resonance peak can be reconstructed, a precise background estimate is crucial for the measurement of Higgs couplings. Needed improvements include NLO QCD corrections, single top quark production backgrounds, the combination of shower Monte Carlo programs with higher order QCD matrix element calculations and more.

(f) Both in the inclusive and WBF analyses any given channel contains a mixture of events from $g g \rightarrow H$ and $q q$ $\rightarrow q q H$ production processes. The determination of this mixture adds another source of systematic uncertainty, which was not included in the present study. In ratios of $X$ observables (or of different $Y_{i}$ ) these uncertainties largely cancel, except for the effects of acceptance variations due to different signal selections. Since an admixture from the wrong production channel is expected at the 10 to $20 \%$ level only, these systematic errors are not expected to be serious.

(g) We have only analyzed the case of a single neutral, $C P$ even Higgs resonance with couplings which are close to the ones predicted in the SM. While this case has many applications, e.g. for the large $m_{A}$ region of the MSSM, more general analyses, in particular of the MSSM case, are warranted and highly promising.

While much additional work is needed, our study clearly shows that the LHC has excellent potential to provide detailed and accurate information on Higgs boson interactions. The observability of the Higgs boson at the LHC has been clearly established, within the SM and extensions like the MSSM. The task now is to sharpen the tools for accurate measurements of Higgs boson properties at the LHC.

\section{ACKNOWLEDGMENTS}

We would like to thank the organizers of the Les Houches Workshop, where this work was initiated, for getting us together in an inspiring atmosphere. Useful discussions with M. Carena, A. Djouadi, K. Jakobs and G. Weiglein are gratefully acknowledged. We thank CERN for the hospitality extended to all of us during various periods of this work. The research of E.R.-W. was partially supported by the Polish Government Grant No. KBN 2P03B14715, and by the Polish-American Maria Sklodowska-Curie Joint Fund II in cooperation with PAA and DOE under Project No. PAA/ DOE-97-316. The work of D.Z. was supported in part by the University of Wisconsin Research Committee with funds granted by the Wisconsin Alumni Research Foundation and in part by the U.S. Department of Energy under Contract No. DE-FG02-95ER40896.
[1] ATLAS Collaboration, ATLAS Detector and Physics Performance Technical Design Report, report CERN/LHCC/99-15 (1999).

[2] G. L. Bayatian et al., CMS Technical Proposal, report CERN/ LHCC/94-38 (1994); D. Denegri, Prospects for Higgs (SM and MSSM) searches at LHC, talk in the Circle Line Tour Series, Fermilab, 1999 (http://www-theory.fnal.gov/CircleLine/ DanielBG.html); R. Kinnunen and D. Denegri, Expected SM/ SUSY Higgs observability in CMS, CMS NOTE 1997/057; R. Kinnunen and A. Nikitenko, Study of $H_{S U S Y} \rightarrow \tau \tau \rightarrow l^{ \pm}+h^{\mp}$ $+E_{t}^{\text {miss }}$ in CMS, CMS TN/97-106; R. Kinnunen and D. Denegri, The $H_{S U S Y} \rightarrow \tau \tau \rightarrow h^{ \pm}+h^{\mp}+X$ channel, its advantages and potential instrumental drawbacks, hep-ph/9907291.

[3] For recent reviews, see, e.g., J. L. Rosner, Comments Nucl. Part. Phys. 22, 205 (1998); K. Hagiwara, Annu. Rev. Nucl. Part. Sci. 48, 463 (1998); W. J. Marciano, hep-ph/9902332, and references therein.

[4] H. E. Haber and R. Hempfling, Phys. Rev. D 48, 4280 (1993); M. Carena, J. R. Espinosa, M. Quiros, and C. E. M. Wagner, Phys. Lett. B 355, 209 (1995); S. Heinemeyer, W. Hollik, and G. Weiglein, Phys. Rev. D 58, 091701 (1998); R.-J. Zhang, Phys. Lett. B 447, 89 (1999).

[5] J. F. Gunion, L. Poggioli, R. Van Kooten, C. Kao, and P. Rowson, hep-ph/9703330.

[6] D. Rainwater and D. Zeppenfeld, J. High Energy Phys. 12, 005 (1997).

[7] D. Rainwater, Ph.D. thesis, hep-ph/9908378.

[8] D. Rainwater, D. Zeppenfeld, and K. Hagiwara, Phys. Rev. D 59, 014037 (1999).
[9] T. Plehn, D. Rainwater, and D. Zeppenfeld, Phys. Rev. D 61, 093005 (2000).

[10] D. Rainwater and D. Zeppenfeld, Phys. Rev. D 60, 113004 (1999); 61, 099901 (2000).

[11] A. Nikitenko, talk given at the workshop on Physics at TeV Colliders, Les Houches, France, 1999.

[12] R. N. Cahn, S. D. Ellis, R. Kleiss, and W. J. Stirling, Phys. Rev. D 35, 1626 (1987); V. Barger, T. Han, and R. J. N. Phillips, ibid. 37, 2005 (1988); R. Kleiss and W. J. Stirling, Phys. Lett. B 200, 193 (1988); D. Froideveaux, in Proceedings of the ECFA Large Hadron Collider Workshop, Aachen, Germany, 1990, edited by G. Jarlskog and D. Rein (CERN Report No. 90-10, Geneva, Switzerland, 1990), Vol. II, p. 444; M. H. Seymour, ibid., p. 557; U. Baur and E. W. N. Glover, Nucl. Phys. B347, 12 (1990); Phys. Lett. B 252, 683 (1990).

[13] V. Barger, K. Cheung, T. Han, and R. J. N. Phillips, Phys. Rev. D 42, 3052 (1990); V. Barger et al., ibid. 44, 1426 (1991); V. Barger, K. Cheung, T. Han, and D. Zeppenfeld, ibid. 44, 2701 (1991); 48, 5444(E) (1993); 48, 5433 (1993); V. Barger et al., ibid. 46, 2028 (1992).

[14] D. Dicus, J. F. Gunion, and R. Vega, Phys. Lett. B 258, 475 (1991); D. Dicus, J. F. Gunion, L. H. Orr, and R. Vega, Nucl. Phys. B377, 31 (1991).

[15] Y. L. Dokshitzer, V. A. Khoze, and S. Troian, in Proceedings of the 6th International Conference on Physics in Collisions, 1986, edited by M. Derrick (World Scientific, Singapore, 1987), p. 365; J. D. Bjorken, Int. J. Mod. Phys. A 7, 4189 (1992); Phys. Rev. D 47, 101 (1993). 
[16] V. Barger, R. J. N. Phillips, and D. Zeppenfeld, Phys. Lett. B 346, 106 (1995).

[17] CMS Collaboration, “The electromagnetic calorimeter project,', Technical Design Report, CERN/LHCC 97-33, CMS TDR 4, 1997.

[18] Katri Lassila-Perini, "Discovery Potential of the Standard Model Higgs in CMS at the LHC,', Diss. ETH N.12961.

[19] I. Iashvili, R. Kinnunen, A. Nikitenko, and D. Denegri, "Study of the $H \rightarrow Z Z^{*} \rightarrow 4 l^{ \pm}$in CMS,'” CMS TN/95-059 (1995).

[20] M. Dittmar and H. Dreiner, Phys. Rev. D 55, 167 (1997); hep-ph/9703401, CMS NOTE 1997/083.

[21] W. Keung and W. J. Marciano, Phys. Rev. D 30, 248 (1984).

[22] V. Barger and R. J. Phillips, Collider Physics, Frontiers in Physics Vol. 71 (Addison-Wesley, Redwood City, CA, 1987).

[23] A. Djouadi, M. Spira, and P. Zerwas, Phys. Lett. B 264, 440 (1991); M. Spira, A. Djouadi, D. Graudenz, and P. M. Zerwas,
Nucl. Phys. B453, 17 (1995).

[24] T. Han, G. Valencia, and S. Willenbrock, Phys. Rev. Lett. 69, 3274 (1992).

[25] M. Carena, S. Mrenna, and C. E. Wagner, Phys. Rev. D 60, 075010 (1999); H. Eberl, K. Hidaka, S. Kraml, W. Majerotto, and Y. Yamada, hep-ph/9912463.

[26] L. J. Hall, R. Rattazzi, and U. Sarid, Phys. Rev. D 50, 7048 (1994); R. Hempfling, ibid. 49, 6168 (1994); M. Carena, M. Olechowski, S. Pokorski, and C. E. Wagner, Nucl. Phys. B426, 269 (1994); D. M. Pierce, J. A. Bagger, K. Matchev, and R. Zhang, ibid. B491, 3 (1997); J. A. Coarasa, R. A. Jimenez, and J. Sola, Phys. Lett. B 389, 312 (1996); R. A. Jimenez and J. Sola, ibid. 389, 53 (1996).

[27] Particle Data Group, C. Caso et al., Eur. Phys. J. C 3, 1 (1998). 